\title{
Kernos
}

Revue internationale et pluridisciplinaire de religion grecque antique

19 | 2006

Varia

\section{S.C. HUMPHREYS, The Strangeness of Gods. Historical Perspectives on the Interpretation of Athenian Religion}

\section{Vinciane Pirenne-Delforge}

\section{(2) OpenEdition}

\section{Journals}

Édition électronique

URL : https://journals.openedition.org/kernos/475

DOI : $10.4000 /$ kernos. 475

ISSN : 2034-7871

\section{Éditeur}

Centre international d'étude de la religion grecque antique

\section{Édition imprimée}

Date de publication : 1 janvier 2006

Pagination : 463-465

ISSN : 0776-3824

\section{Référence électronique}

Vinciane Pirenne-Delforge, « s.c. HuMPHREYs, The Strangeness of Gods. Historical Perspectives on the Interpretation of Athenian Religion », Kernos [En ligne], 19 | 2006, mis en ligne le 22 mars 2011, consulté le 24 août 2022. URL : http://journals.openedition.org/kernos/475 ; DOI : https://doi.org/ 10.4000/kernos.475 
peut paraître encore inchoatif, mais il mérite d'être salué. On se félicitera aussi du recours fréquent aux œuvres poétiques. C'est qu'artistes et poètes ont joué un rôle décisif dans la transmission des traditions religieuses et dans l'apprentissage d'un langage symbolique. Aussi bien l'expérience religieuse et la quête du beau sont-elles, chez les Grecs, en connexion très étroite. Que l'on songe, par exemple, à leur appréhension poétique de la nature environnante ou à la place que tient la musique dans les célébrations festives auxquelles les dieux sont censés participer et dont ils offrent eux-mêmes le modèle aux hommes. La dimension esthétique de l'expérience religieuse aurait mérité peut-être de faire l'objet d'un développement un tant soit peu circonstanciée : jouer de la flûte ou de la cithare, composer et chanter des hymnes, former des chœurs et danser sont aussi, pour les Grecs, une manière habituelle d'entrer en relation avec le divin et de le célébrer. Or c'est en vain que, dans l'index, on chercherait même le nom des Muses.

L'ouvrage comporte de fait un index très complet des matières traitées qui fait suite à une liste des figures (dont une carte des sanctuaires évoqués), à un index des auteurs et des textes cités ainsi qu'à une bibliographie sélective qui suit l'ordre des chapitres. Ces compléments contribuent à faire de l'ouvrage un outil précieux. Mais il est bien davantage. En faisant place aux représentations mentales ainsi qu'aux émotions qui affectent les groupes et les individus, il fait des pratiques religieuses une présentation in vivo qui est de nature à faire comprendre que la religion des Grecs pouvait être autre chose que la simple répétition mécanique de gestes réputés pieux. C'est là une avancée qui n'est pas mince et à propos de laquelle on lira avec profit les propos nuancés de l'A. (p. viII). Pour cette raison, de même que pour la qualité de son information, le livre est à recommander particulièrement aux incipientes, mais il ne manquera pas d'intéresser aussi les progredientes dans l'étude de la religion grecque et rendra efficacement service à ceux qui ont mission de l'enseigner.

André Motte

(Université de Liège)

S.C. Humphreys, The Strangeness of Gods. Historical Perspectives on the Interpretation of Athenian Religion, Oxford, University Press, 2004. 1 vol. $14,5 \times 22 \mathrm{~cm}, 399$ p. ISBN : 0-19-926923-8.

Ce livre résiste vigoureusement à l'exercice du résumé d'un tout cohérent même s'il ne s'agit pas d'un ouvrage collectif. Les six chapitres qui le composent, dont quatre trouvent leur origine dans des articles déjà publiés, se succèdent sans lien thématique ferme. L'A. le reconnaît à demi-mots dès l'introduction : "The order of the chapters here is that of ring-composition rather than linear development of an argument» (p. 4). Un arrière-plan fédérateur est le souci de rendre explicite la tension que tous les interprètes des religions antiques connaissent entre leurs déterminismes culturels plus ou moins conscients et le désir puissant de se mettre à l'écoute de sources censées parler d'elles-mêmes, sans filtre. Et s'il est possible de mettre au jour un thème fédérateur, c'est celui du changement en matière religieuse, contre la vision canonique du « conservatisme » supposé des pratiques qui légitime trop facilement des reconstitutions statiques et intemporelles.

Le chapitre 1 (Classics and Colonialism: towards an erotics of the discipline, 2002) a une portée historiographique et propose une réflexion théorique ardue. Il met en perspective l'institutionnalisation de l'enseignement des lettres classiques, avec tous les prismes déformants qu'impliquait cette «colonisation » du passé. La réflexion ainsi menée est complexe et aboutit à une série de remarques sur l'enseignement des textes 
classiques, non dans leur prétendue intemporalité esthétisante, mais bien en tant que productions complexes d'une société très différente de la nôtre et dont la lecture interactive devrait idéalement donner aux étudiants les outils d'une véritable critique.

Le chapitre 2 (Dynamics of the Greek 'Breakthrough': the dialogue between philosophy and religion, 1986) se penche - sans le dire explicitement en ces termes, que l'on ne trouve qu'au chap. 1 (p. 23, 48) - sur la question du passage du muthos au logos. Le discours polémique et rationalisant du $\mathrm{v}^{\mathrm{e}} \mathrm{s}$. av. J.-C. n'aurait pas relégué le religieux dans le seul registre de la tradition ou de la vie privée. C'est un véritable dialogue qui se serait instauré entre rationnel et irrationnel, entre deux visions du monde, dont la première était à la fois empirique (processus d'observation), politique (primauté de la vie publique) et logique (raisonnement et argumentation). Poésie et pensée religieuse ainsi attaquées auraient dès lors répondu par une conception du divin plus abstraite e.g. les abstractions divinisées -, par une modernisation des actes rituels - e.g. des refontes de calendriers - et par le développement de doctrines religieuses 'scientifiques' (orphisme, astrologie, magie...). La problématique abordée est certes difficile, mais la lecture qui est faite de certains textes est surprenante: peut-on vraiment interpréter $I G$ II 337 (l'autorisation donnée aux gens de Kition d'acheter un terrain pour édifier un sanctuaire de leur Aphrodite, comme les Égyptiens l'avaient fait pour Isis avant eux) comme une indication d'un " growing taste for the exotic in religious ritual, a feeling that only what was wild, strange, and altogether different from ordinary life was truly religious » (p. 73) ? L'exemple ne soutient guère une telle affirmation. Il n'empêche que nous n'avons guère le choix : nous sommes tous contraints d'utiliser des sources d'information éclatées et de nature très différente afin de donner une certaine épaisseur historique à la société que l'on étudie. Mais les sources ne parlent pas d'elles-mêmes et la voix que l'interprète leur donne risque souvent d'être la sienne. La tension que l'introduction du livre évoquait est tout particulièrement présente ici et la portée des changements mis au jour parfois difficilement estimable.

Le chapitre 3 (Lycurgus of Boutadai: an Athenian aristocrat, 1985) est un portrait remarquablement informé de l'homme politique athénien, membre de l'un des plus fameux genè d'Athènes et réformateur éclairé, notamment en matière de religion. On retrouve donc ici le thème du changement religieux. Le texte de l'article de 1985 est suivi d'un Afterword. C'était le cas déjà pour les deux premiers chapitres. Mais alors que ceux-là se contentaient d'une mise à jour bibliographique, celui-ci est un nouveau développement sur les innovations religieuses de la période de Lycurgue et une réévaluation de son style politique et de son idéologie. Quelles que soient les qualités de ces deux essais juxtaposés, pourquoi n'avoir pas profité de cette nouvelle publication - un livre à une voix de surcroît -, pour livrer un chapitre cohérent, l'étude initiale étant refondue, réélaborée et mise à jour?

Le chapitre 4 ( $A$ Sense of Agency: religion in the Attic demes) est une étude inédite qui se penche notamment sur ces documents remarquables que sont les «calendriers " des dèmes. L'A. tente d'y déceler les nouveautés intervenues entre 507, moment de la création des dèmes, et 307. Le rapport avec la cité est tout particulièrement étudié et l'image qui ressort de l'analyse est celle d'une vie rurale dynamique, en dialogue créatif avec la vie civique et ses institutions. Reste un problème de méthode : hormis pour les questions de finances religieuses, l'évolution mise au jour n'est pas décelable dans les calendriers étudiés. Elle est le fruit d'hypothèses indépendantes d'eux et juxtaposées à la description de leur contenu (c'est tout particulièrement le cas pour le calendrier de Thorikos [p. 156-165] et la conclusion de la page 194 sur les trois 
périodes des relations entre dèmes et cité) $)^{2}$. Certes, il est des documents qui font voir le changement : l'inscription du serment des éphèbes mise au jour à Acharnes manifeste l'adhésion du dème à la réforme éphébique du temps de Lycurgue. Mais on adhère à l'affirmation que l'A. livre à la fin de son chapitre : «It is, of course, characteristic of the state of the evidence for Greek religion that interpretation have to be based on controversial readings of fragmentary and problematic texts » (p. 193). Il reste à être inventif - et prudent - dans les interpretive frameworks à leur appliquer.

Le chapitre 5 (Historicizing Fertility - dont l'Afterword laisse entendre qu'il avait déjà été publié mais dont l'original n'est pas daté) revient sur des problèmes d'historiographie et de déterminisme culturel. L'A. argumente avec une grande force de conviction et une érudition remarquablement maîtrisée. Elle démontre ainsi que la vision canonique du motif de la fertilité/fécondité comme noyau dur des rituels, hérité du passé, est une élaboration moderne, à l'instar des filtres de lecture dénoncés dans le premier chapitre. C'est l'historicisme qui est ici mis en cause et son avenir interrogé.

Le chapitre 6 (Metamorphoses of Tradition: the Athenian Anthesteria) s'ouvre sur l'affirmation que les rituels changent ${ }^{3}$. Ce doit donc être le cas aussi pour les Anthestéries athéniennes. Notre image de cette fête, qui court sur trois journées du mois de mars, est une sorte de puzzle fait de pièces et fragments de tradition épars et chronologiquement incohérents. Encore un bel exemple de ce problématique state of the evidence... Mais ces pièces et morceaux pourraient raconter une véritable histoire plutôt que de fournir un cliché figé et intemporel. C'est cette histoire de longue durée (depuis la migration ionienne jusqu'à Photios!) qui est ici reconstituée, avec une grande finesse d'analyse, et une part inévitable de conjecture, dont la conclusion du chapitre montre la pleine conscience que l'A. en a.

Une vaste bibliographie et de très bons index font de ce livre assez déroutant dans sa conception un excellent instrument de travail sur les innombrables mises au point de détail qui abondent au fil des pages. Quant à la lecture suivie, d'un bout à l'autre, elle permet de voir se nouer les perspectives historiques du sous-titre, mais les dieux du titre principal, sans doute en raison de leur « étrangeté », restent largement hors du champ de l'investigation

Vinciane Pirenne-Delforge (FNRS - Université de Liège)

Pierre Eluinger, La fin des maux. D'un Pausanias à l'autre. Essai de mythologie et d'bistoire, Paris, Les Belles Lettres, 2005. 1 vol. $15 \times 22 \mathrm{~cm}, 374$ p. (Coll. Histoire). ISBN : 2-251-38073-6.

J'aimerais rompre avec l'ouverture généralement convenue des comptes rendus académiques et dire d'emblée que ce livre m'a enthousiasmée. Je l'ai lu d'un bout à l'autre, quasiment sans interruption, tant le rythme et l'intrigue en sont remarquablement menées. Il n'est sans doute pas inutile de préciser que, plongée à d'autres fins dans l'œuvre de Pausanias le Périégète, j'ai trouvé dans cette lecture une sorte de résonance avec mes propres interrogations. Cette communauté d'intérêt, même si elle ne s'assortit pas systématiquement d'une communauté de vue, n'a pas peu compté

\footnotetext{
${ }^{2}$ Un détail : les restitutions de $I G \mathrm{II}^{2}$ 1358A, 1. 13-19 proposées à la page 169 font surgir des enagismata que la thèse de G. Ekroth (Kernos, suppl. 12, 2002) rend à mon sens problématiques.

${ }^{3}$ Le thème est d'actualité. Une série de séminaires ont été donnés à l'Université de Heidelberg sur le thème Dynamics of ritual. Ces textes constituent le $16^{\mathrm{e}}$ supplément de Kernos, sous le titre Ritual and Communication in the Graeco-Roman World (éd. par E. Stavrianopoulou).
} 